\title{
Fernreisen
}

\section{Umfassend geimpft, entspannt im Urlaub}

Die häufigsten impfpräventablen Infektionen auf Reisen sind ETEC-Gastroenteritiden sowie Influenza und Typhus. Auch der Tollwutschutz ist bei Fernreisen zu empfehlen.

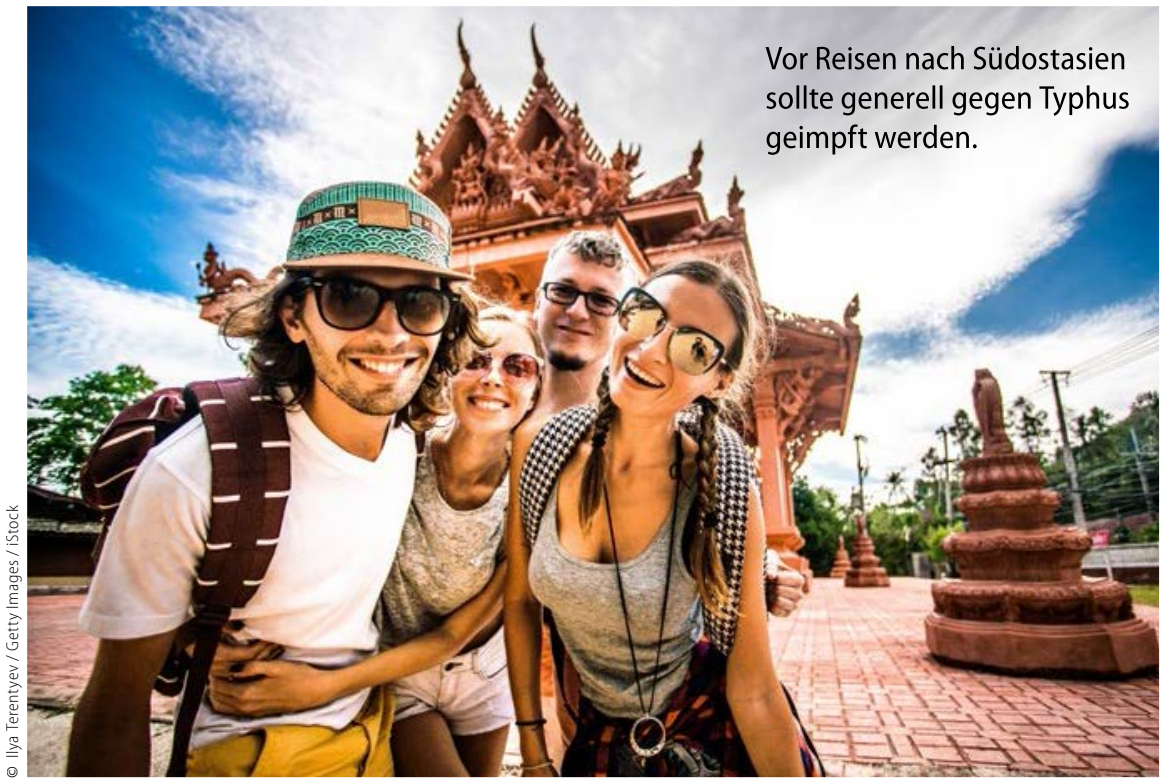

Eine aktuelle Analyse der Häufigkeit impfpräventabler Reise-Infektionen hat Prof. Dr. Tomas Jelinek vom Berliner Centrum für Reisemedizin (BCRM) bei einer Veranstaltung in Mannheim vorgestellt [1]. Danach kommt es pro Monat Aufenthalt in den Tropen oder Subtropen bei etwa einem von 30 Reisenden zu einer Gastroenteritis durch enterotoxische E. coli (ETEC), bei einem von 100 Reisenden $\mathrm{zu}$ einer Influenza, bei einem von 3000 Reisenden zu Typhus sowie bei einem von 8000 Reisenden zu einer Hepatitis A. Die Infektionen mit Typhus stammen dabei allesamt aus Südostasien.

Auch im Vorfeld von Reisen gilt die Empfehlung der Impfung gegen Influenza vor allem für Menschen mit erhöhtem Risiko für Komplikationen, wie Senioren oder chronisch Kranke. Auf Kreuzfahrtschiffen ist das Infektionsrisiko durch das dichte Zusammenleben erhöht.
Gegen ETEC-Enteritis empfiehlt Jelinek die Schluckimpfung gegen Cholera (Dukoral $\left.{ }^{\circledast}\right)$. Nach Studiendaten schützt dieser Impfstoff aufgrund einer Kreuzprotektion auch gegen ETEC. Bis zu 40\% der Reisedurchfälle ließen sich damit verhindern, so der Reisemediziner in Mannheim. Der Impfstoff ist jedoch nur in der Schweiz und nicht in Deutschland zur Prävention von ETEC-Infektionen zugelassen. Er muss hierzulande also off-label gegeben werden.

„In Südostasien hat Typhus inzwischen Hepatitis A bei der Inzidenz überholt“, berichtete Jelinek weiter. Weil schon eine sehr geringe Menge von Keimen für die Infektion ausreicht, sollte bei Reisen in die Region generell gegen Typhus geimpft werden - und zwar "auch bei dem Manager, der nur schnell in Neu-Delhi im Hilton-Hotel absteigen will“. Immerhin liege die Schutzwirkung sowohl bei den parenteralen als auch bei den oralen Impfstoffen bei 60-70\%. Gute Erfahrungen hat Jelinek hier auch mit der Schutzimpfung gegen Hepatitis A plus Typhus in einer Spritze gemacht. Diese Kombinationsimpfstoffe sind allerdings zurzeit in Deutschland nicht verfügbar, weshalb auf die Mono-Impfstoffe zurückgegriffen werden muss.

Schließlich rät Jelinek bei Fernreisen auch zum Tollwutschutz. Die stets tödliche Infektion ist zwar sehr selten, bei 20100 Reisenden kommt es jedoch zu Tierbissen mit potenziellem Tollwutrisiko. „Hierzu haben wir jede Woche Anfragen“, so der Reisemediziner. Für die Impfung ist auch ein Schema zur Schnellimmunisierung in Sicht, bei dem an den Tagen 0-3-7 geimpft wird.

\section{Basisimpfschutz überprüfen!}

Reisende sind meist Erwachsene, die sonst nur selten zum Arzt gehen. Bei einer reisemedizinischen Beratung sollte daher stets auch der Basisimpfschutz überprüft und Impflücken etwa gegen Masern, Polio, Diphtherie, Tetanus und Pertussis geschlossen werden.

Vor allem auch der Masernschutz ist auf vielen Reisen zu empfehlen. Die Erkrankungszahlen nehmen weltweit in vielen Regionen zu. So wurden etwa in Singapur von Januar bis Mitte Mai 50 Fälle gemeldet, während es im selben Zeitraum des Vorjahres nur 17 waren, berichtet die International Society für Infectious Diseases (ISID). In Neuseeland gab es Anfang Mai einen Ausbruch an einem College in der Region Waikato mit 20 Fällen und Ausbreitung in andere Regionen.

Eine Änderung des Impfschemas gibt es bei der Gelbfieber-Impfung. Die WHO rät nicht mehr zu Booster-Impfungen alle zehn Jahre. Es gilt: Eine einzige Impfung reicht für lebenslangen Schutz.

(Wolfgang Geissel)

1. J Trav Med. 2015;22:1-12 